\title{
Soliton dynamics of an atomic spinor condensate on a Ring Lattice
}

\author{
Indubala I. Satija ${ }^{1}$, Carlos L. Pando L. ${ }^{2}$ and Eite Tiesinga ${ }^{3}$ \\ ${ }^{1}$ School of Physics, Astronomy and Computational Sciences, George Mason University, Fairfax, VA 22030, USA \\ ${ }^{2}$ IFUAP, Universidad Autonoma de Puebla, Apdo, Postal J-48, Puebla 72570, Mexico and \\ ${ }^{3}$ Joint Quantum Institute, National Institute of Standards and Technology and the University of Maryland, \\ 100 Bureau Drive, Stop 8423, Gaithersburg, MD 20899-8423, USA
}

(Dated: May 28, 2022)

\begin{abstract}
We study the dynamics of macroscopically-coherent matter waves of an ultra-cold atomic spin-1 or spinor condensate on a ring lattice of six sites and demonstrate a novel type of spatio-temporal internal Josephson effect. Using a discrete solitary mode of uncoupled spin components as an initial condition, the time evolution of this many-body system is found to be characterized by two dominant frequencies leading to quasiperiodic dynamics at various sites. The dynamics of spatially-averaged and spin-averaged degrees of freedom, however, is periodic enabling an unique identification of the two frequencies. By increasing the spin-dependent atomatom interaction strength we observe a resonance state, where the ratio of the two frequencies is a characteristic integer multiple and the spin-and-spatial degrees of freedom oscillate in "unison". Crucially, this resonant state is found to signal the onset to chaotic dynamics characterized by a broad band spectrum. In a ferromagnetic spinor condensate with attractive spin-dependent interactions, the resonance is accompanied by a transition from oscillatory- to rotational-type dynamics as the time evolution of the relative phase of the matter wave of the individual spin projections changes from bounded to unbounded.
\end{abstract}

PACS numbers: 03.75.Ss,03.75.Mn,42.50.Lc, 73.43.Nq

\section{INTRODUCTION}

Spinor condensates are atomic Bose-Einstein condensates (BEC) with an internal spin degree of freedom that combine magnetism with condensation. Examples are opticallytrapped spinor condensates of atomic rubidium-87[1-4], sodium [5, 6], or chromium [7] with either spin or angular momentum $f=1,2$, or 3 condensates with a three-, five-, or seven-component vector order parameter. In contrast to mixtures of two or more atomic states [8] or mixtures of several atomic species, spin-changing collisions in spinor condensates permit coherent dynamics among the hyperfine states. In a typical process for two $f=1$ atoms, one in spin component $m=-1$ and one in $m=+1$, can reversibly scatter into two atoms with spin component $m=0$, which conserves the global magnetization of the condensate. This coherent spin mixing leads, nevertheless, to oscillations of the spin populations, and is an analogue of Josephson oscillations in ultracold atoms [9-13]. Hence, just as collisional interactions allow for a single-component condensate to be spatially coherent, spin-changing collisions, driven by internal interactions, allow coherence among internal degrees of freedom. A positive or negative sign of the strength of the spin-changing interaction determines whether the systems behave anti-ferromagnetic or ferromagnetic, respectively [10, 14]. Spinor physics has also been studied in optical lattices with exactly two atoms per lattice site [15].

In view of the nonlinear nature due to interparticle interactions and high degree of control in the experiments, BEC systems are ideal systems for visualizing a wide variety of nonlinear phenomena. This includes solitary waves, the localized nonlinear traveling waves that retain their shape, size and speed during propagation [16]. Experimental realization of solitons in a homogeneous single component BEC systems is a hallmark of the quantum coherence associated with many body systems.[17.-19] There has also been theoretical studies of solitons in a homogeneous spin- 1 condensate [20, 21]. Furthermore, theoretical and experimental studies of double-well bosonic Josephson junctions have unveiled novel phenomena such as broken symmetry macroscopic quantum self-trapping [22] and $\pi$-modes [23-25] as well as symmetry restored swapping modes [22]. In addition, numerical investigation of BEC systems, spatially separated into a ring lattice have demonstrated chaotic dynamics, deterministic dynamics with sensitive dependence on initial conditions. [26-[28]

In this paper, we explore the quantum coherent time evolution of a spin-1 BEC that is spatially separated into six weakly-coupled sites arranged to form a ring geometry, see Fig. 11. For a large number of atoms such a system is well represented by a three-component wavefunction or order parameter, $\vec{\Phi}(\vec{x}, t)$, that satisfies a nonlinear Gross-Pitaevskii equation [9, 10]. Here, we restrict our calculations to the case with no external magnetic field. More importantly, we assume that all sites and spin components have at all times the same identical localized spatial-mode function, $\phi(\vec{x})$. That is, for the $m^{\text {th }}$-component of $\vec{\Phi}(\vec{x}, t)$ we have

$$
\Phi_{m}(\vec{x}, t)=\sum_{n=1}^{L} \psi_{n}^{m}(t) \phi\left(\vec{x}-\vec{x}_{n}\right),
$$

where $L$ is the number of sites, $\vec{x}_{n}$ is the center of site $n$, and the dimensionless $\psi_{n}^{m}(t)$ are complex time-dependent amplitudes. The overlap between spatial-mode functions at different sites leads to tunneling between neighboring sites.

More advanced numerical modeling uses time varying mode functions [29, 30] or directly solves for the threedimensional Gross-Pitaevskii equation[18]. As a first study in the soliton dynamics of a spinor condensate we believe that our simplified approach is justified. It shows the generalization of the spinor Josephson effect as well as the route to chaos 

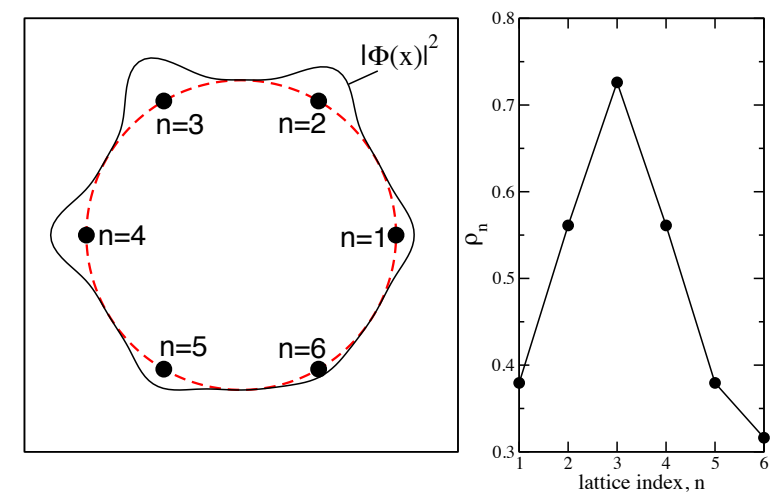

FIG. 1: (color online) Panel a) shows a schematic of a ring lattice of six sites (red dashed circle with labeled markers) containing a $f=$ 1 spinor condensate. The solid black line represents the superfluid population along the ring as a polar graph. Panel b) shows the initial fractional population, $\rho_{n}$, as a function of site index $n$ used in our dynamical simulations. The distribution corresponds to an excited (period-6) solution of the DNLSE. For each site the population of the three magnetic sublevels of the spinor is the same.

in its clearest form. It should be noted that the ring lattice with less than three sites is an integrable system. BEC on a lattice with three or more than three sites exhibits amazing degree of complexity including coexistence of regular and irregular behavior that is known to accompany chaotic dynamics. In our detailed numerical exploration of ring lattices with different numbers of sites, the key characteristics of the time evolution as discussed in this paper are independent of the number of sites. Here, we will present our results for a ring lattice of six sites only.

Our initial state is a discrete solitary wave that is an excited eigenstate of the discrete nonlinear Schrödinger equation (DNLSE) satisfied by the $\psi_{n}^{m}(t)$ in the absence of spinchanging interactions. The spin-changing interaction then induces oscillatory dynamics, an internal Josephson effect where different spin components evolve with different amplitudes and phases. In reality the spin-changing Hamiltonian can not be turned off and this state needs to be engineered by a combination of resonant electro-magnetic radiation that controllably creates superpositions of $m$-states [6] and offresonant spatially-dependent light forces that induce solitons [17, 18].

In this article we show that for weak spin-changing interactions the dynamics on the ring lattice is dominated by two frequencies. Interestingly, one of these frequencies, which we denote as $\omega$, describes the periodic behavior of spatiallyaveraged spin populations. The second frequency, denoted by $\Omega$, describes the dynamics of the site-dependent population summed over the three spin components. In other words, the dynamics of collective or averaged coordinates is pendulumlike. As we discuss below, this is somewhat surprising as the spatial profiles of the three spin components evolve differently and the dynamics on the ring should deviate from that for a "simple" trapped spinor condensate. The single-mode approximation for the latter system has been shown to lead to pendulum-type physics [10, 12]. Our study suggests that the origin of this "regular" global dynamics has its roots in the strong correlations among different lattice sites and spin projections and is not rooted in thermal averaging over many degrees of freedom. We note that $\omega$ is solely determined by the spin-changing interaction, whereas $\Omega$ shows a rather weak dependence. We find that $\Omega$ is mainly controlled by the size of the ring.

By increasing the absolute value of the spin-dependent interaction strength, the two frequencies can be mode locked. This resonance condition is found to describe the onset to chaotic dynamics. In other words, the spinor condensate exhibits a transition from quasi-periodic dynamics to chaotic dynamics where at the onset to transition, all local and global degrees of freedom oscillate in unison. Consequently, the initial soliton profile reappears periodically, providing a unique demonstration of a novel-type of spatio-temporal internal Josephson effect. Furthermore, in contrast to the antiferromagnetic case, in the ferromagnetic condensate, the resonance is accompanied by a transition from bounded to unbounded dynamics. In the anti-ferromagnetic case, the dynamics remains bounded and oscillatory. Nevertheless, beyond the resonance additional frequencies appear.

For sake of simplicity, we restrict our simulations to zero magnetization. This is enforced by choosing initial conditions where the $m=+1$ and $m=-1$ components have same initial wave function. In fact, we choose an initial state where all three components have the same initial wave function. An alternate initial state with zero magnetization and contains mostly $m= \pm 1$ atoms lead to similar results. It will not be described here.

In section $\Pi$ we review the mean-field equations that describe the evolution of the spinor condensate on a lattice as well as the underlying single-mode approximation approximation on each lattice site. The latter has been shown to provide a reasonable description for the continuum system. For our ring lattice, the SMA suggests collective coordinates to describe the global dynamics. The initial state is described in Sec. III] In section IV] we show numerical simulations for weak positive, anti-ferromagnetic spin-dependent integrations and show that the dynamics, although complex, is dominated by two frequencies. In section $\mathrm{V}$, we discuss the resonance condition that occurs when the spin-dependent interaction strength is increased. At resonance we observe an onset to chaos. Section VI briefly illustrates the ferromagnetic case. We conclude in Sec. VII.

\section{MEAN-FIELD EQUATIONS FOR A SPINOR CONDENSATE AND COLLECTIVE COORDINATES}

An atomic spinor condensate with large atom number is described within mean-field theory by a complex vector order parameter or condensate wave function $\Psi(\vec{x}, t)$, whose evolution is governed by a multi-component Gross-Pitaevskii equation [9, 10]. We will assume that the order parameter can be approximated by Eq. 1. where a single time-independent mode function, $\phi(\vec{x})$, determines the spatial dependence in 
each well. We denote this by the $L$-site single-mode approximation (L-SMA) in analogy to the single-mode approximation (SMA) for a spinor condensate in a dipole trap.

The interactions between two spin-1 atoms have a spin-independent and a spin-dependent or (spinchanging) contribution. Within a mean-field theory and the L-SMA their strength is given by $c_{q}=4 \pi \hbar^{2} g_{q} /(2 \mu) \int d \vec{x}|\phi(\vec{x})|^{4} / \int d \vec{x}|\phi(\vec{x})|^{2}$ with $q=0$ and 2 for the spin-independent and spin-dependent contribution, respectively. Here $\mu$ is the reduced mass for two atoms and $\hbar$ is the reduced Planck constant. The lengths $g_{q}$ are $g_{0}=\left(a_{0}+2 a_{2}\right) / 3$ and $g_{2}=\left(a_{2}-a_{0}\right) / 3$, where $a_{0}$ and $a_{2}$ are scattering lengths for $s$-wave collisions of two $f=1$ bosons with total angular momentum $F=0$ and 2, respectively. For stable condensed gases we require that $c_{0}>0$. We note that $c_{2}>0$ for anti-ferromagnetic $\mathrm{Na}$ and $c_{2}<0$ for ferromagnetic ${ }^{87} \mathrm{Rb}$.

The dynamics of the $\psi_{n}^{m}(t)$ are governed by the DNLSE

$$
\begin{aligned}
i \hbar \dot{\psi}_{n}^{-1}=- & J\left(\psi_{n-1}^{-1}+\psi_{n+1}^{-1}\right) \\
& +\left(c_{0}+c_{2}\right)\left(\left|\psi_{n}^{-1}\right|^{2}+\left|\psi_{n}^{0}\right|^{2}\right) \psi_{n}^{-1} \\
& +\left(c_{0}-c_{2}\right)\left|\psi_{n}^{1}\right|^{2} \psi_{n}^{-1}+c_{2}\left(\psi_{n}^{0}\right)^{2}\left(\psi_{n}^{1}\right)^{*} \\
i \hbar \dot{\psi}_{n}^{0}=- & J\left(\psi_{n-1}^{0}+\psi_{n+1}^{0}\right)+c_{0}\left|\psi_{n}^{0}\right|^{2} \psi_{n}^{0} \\
+ & \left(c_{0}+c_{2}\right)\left(\left|\psi_{n}^{1}\right|^{2}+\left|\psi_{n}^{-1}\right|^{2}\right) \psi_{n}^{0}+2 c_{2} \psi_{n}^{1} \psi_{n}^{-1}\left(\psi_{n}^{0}\right)^{*} \\
i \hbar \dot{\psi}_{n}^{1}=- & J\left(\psi_{n-1}^{1}+\psi_{n+1}^{1}\right)+\left(c_{0}+c_{2}\right)\left(\left|\psi_{n}^{1}\right|^{2}+\left|\psi_{n}^{0}\right|^{2}\right) \psi_{n}^{1} \\
& +\left(c_{0}-c_{2}\right)\left|\psi_{n}^{-1}\right|^{2} \psi_{n}^{1}+c_{2}\left(\psi_{n}^{0}\right)^{2}\left(\psi_{n}^{-1}\right)^{*}
\end{aligned}
$$

where $J$ is the positive site-to-site tunneling energy and we use periodic boundary conditions. In the absence of the last term on the right hand side of Eqs. 2, 4, the set of equations describes a three-species condensate. Spin-changing terms make a spinor condensate unique as they induce population oscillations between $m$ levels. Equations 244 conserve total atom number and magnetization. In other words, $\sum_{n m}\left|\psi_{n}^{m}(t)\right|^{2}$ and $\sum_{n m} m\left|\psi_{n}^{m}(t)\right|^{2}$ are conserved.

We will monitor the local population of each spin state as well as global or collective coordinates, such as spatially- and spin-averaged population and phases. It is therefore convenient to define $\psi_{n}^{m}(t)=\sqrt{\rho_{n}^{m}(t)} \exp \left[i \phi_{n}^{m}(t)\right]$ with populations $\rho_{n}^{m}(t)$ and phases $\phi_{n}^{m}(t)$. Following Refs. [9, 10] for a single-mode spinor condensate natural local canonical coordinates are

$$
Z_{n}=\rho_{n}^{0}-\left(\rho_{n}^{+1}+\rho_{n}^{-1}\right) \quad \text { and } \quad \gamma_{n}=\phi_{n}^{-1}+\phi_{n}^{1}-2 \phi_{n}^{0} .
$$

as well as globally-averaged coordinates $Z=\sum_{n} Z_{n} / L$ and $\gamma=\sum_{n} \gamma_{n} / L$. Throughout this article we call $\gamma_{n}$ and $\gamma$ spinor phases. Other useful population averages are

$$
\rho_{m}=\frac{1}{L} \sum_{n=1}^{L} \rho_{n}^{m} \quad \text { and } \quad \sigma_{n}=\frac{1}{3} \sum_{m=-1}^{1} \rho_{n}^{m} .
$$

In a dipole trap, or equivalently for $L=1$, a simple spinor model is given by the SMA. The canonical variables $Z$ and $\gamma$ then satisfy the pair of equations

$$
\dot{Z}=\frac{c_{2}}{\hbar}\left(1-Z^{2}\right) \sin \gamma \quad \text { and } \quad \dot{\gamma}=-2 \frac{c_{2}}{\hbar} Z(1+\cos \gamma),
$$

which are independent of the spin-independent interaction with strength $c_{0}$. For small $Z$ and $\gamma$ the dynamics are harmonic. In general, however, these coupled equations describe a nonlinear pendulum whose length depends upon the momentum.

For a $L>2$ ring of lattice sites the collective variables $Z$ and $\gamma$ satisfy

$$
\begin{aligned}
& \dot{Z}=\frac{c_{2}}{\hbar} \sum_{n}\left(\sigma_{n}^{2}-Z_{n}^{2}\right) \sin \gamma_{n} \\
& \dot{\gamma}=-2 \frac{c_{2}}{\hbar} \sum_{n} Z_{n}\left(1+\cos \gamma_{n}\right)+\frac{J}{\hbar} W
\end{aligned}
$$

where $W=\operatorname{Re}\left[\sum_{n}\left(2 \psi_{n+1}^{0} / \psi_{n}^{0}-\psi_{n+1}^{+1} / \psi_{n}^{+1}-\psi_{n+1}^{-1} / \psi_{n}^{-1}\right)\right]$. The spin-independent interaction strength $c_{0}$ does not explicitly appear in these equations. As already mentioned in the introduction and further discussed in next section, this suggests that the spatially-averaged $Z$ and $\gamma$ will exhibit periodic oscillations, with frequency $\omega$, that are solely determined by the spin-changing interaction. Finally, the spin-averaged populations at each site satisfy

$$
\dot{\sigma}_{n}=-\frac{J}{\hbar} \operatorname{Im}\left(\sum_{m=-1}^{1}\left(\psi_{n}^{m}\right)^{*}\left[\psi_{n+1}^{m}+\psi_{n-1}^{m}\right]\right),
$$

which does not explicitly depend on $c_{2}$. Hence, we expect that the $\sigma_{n}$ oscillate periodically, characterized by frequency $\Omega$ and its harmonics.

\section{INITIAL DISCRETE SOLITON}

For a ring lattice of six sites, we use the initial condition shown in Fig. 1. Commonly referred to as a discrete soliton, it is a stationary solution of the DNLSE in the absence of the spin-changing interaction [26, 31] (i.e. with $c_{2}=0$ ) and $c_{0}=$ $J$. Along with phase $\phi_{n}^{m}=0$ or $\pi$ for even or odd site index $n$, respectively, the initial state populations are the same for the three components.

This stationary solution, corresponding to a solution $\psi_{n}^{m}(t)=\psi_{n}^{m}(t=0) \exp (-i \mu t / \hbar)$ where $\mu$ is the chemical potential, is obtained by numerically solving the resulting nonlinear map, as Eqs. 244 reduce to a set of $L$ twodimensional cubic maps. Following Ref. [28], we find that for $L=6$ the solutions are 6 -fold degenerate and for a range of $\mu$ the localized soliton mode in Fig. 1] is the only stable solution. In fact, we have used $\mu=2.5 \mathrm{~J}$. Intriguingly, for these values of the chemical potential the homogeneous solution with the same density at all sites is unstable.

It should also be noted that known localized soliton-type solutions with zero phase for all sites correspond to attractive spin-independent interactions with $c_{0}<0$. To obtain localized solutions for repulsive interactions, one needs to consider solutions with phases different from zero. In general, it can be shown that for a lattice with even number of sites, the mapping $\psi_{n}^{m} \rightarrow(-1)^{n}\left(\psi_{n}^{m}\right)^{*}$ relates a soliton solution for attractive interactions with those with repulsive interactions. For a singlecomponent condensate discrete solitons have been studied extensively for ring lattices of various sizes [26-28]. 


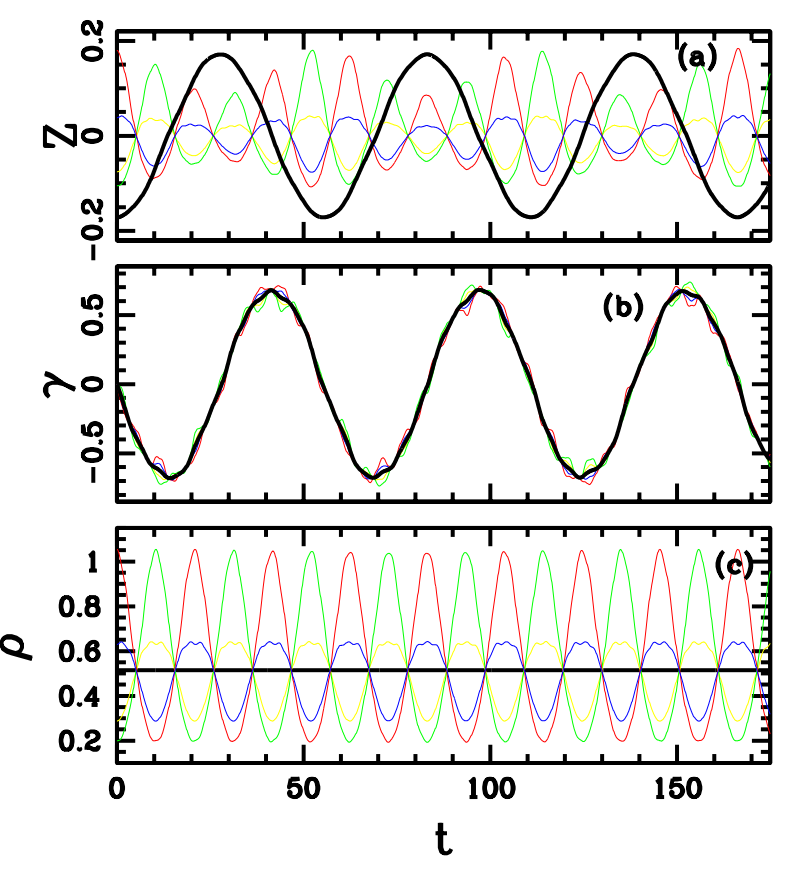

FIG. 2: (color online) Time dynamics of an anti-ferromagnetic spinor soliton on a six-site ring for several spin- and/or spatially-averaged degrees of freedom assuming a small positive spin-changing interaction energy. Time is in units of $\hbar / J$, where $J$ is the tunneling energy between the sites. Calculations are performed for $c_{2}=0.1 \mathrm{~J}$ and $c_{0}=J$. Panel a) shows the dynamics of the spatially-averaged spinor population $Z$ (black line) and the spinor population $Z_{n}$ for the individual sites $n$ (colored lines). The symmetry of the initial soliton around $n=3$ implies that only four of the six sites have a distinct time evolution. Panel b) shows the spinor phases $\gamma$ (black line) and $\gamma_{n}$ (colored lines). Finally, panel c) shows the site-specific population $\sigma_{n}$, averaged over the three spin components.

Experiments with single-component Bose condensates in double-well potentials [24] have observed Josephson oscillations and quantum self-trapping in the limit $J \ll c_{0}$. The opposite limit can also be reached leading to tunneling of (nearly-)independent atoms. Here, we chose a compromise with $c_{0}=J$. As an aside we note that with our initial state and $c_{0}=J$ we have implicitly specified the chemical potential and thus atom number in each site.

\section{QUASIPERIODIC DYNAMICS OF AN ANTI-FERROMAGNETIC SPINOR}

Figure 2 shows the time evolution of the spinor soliton on a six-site ring, described in terms of the spinor coordinates $Z_{n}$ and $Z$, spinor phases $\gamma_{n}$ and $\gamma$, as well as populations $\sigma_{n}$. We use a small positive spin-changing interaction strength $c_{2}$ for an anti-ferromagnetic spinor. We observe that the time dependence of $Z$ and $\gamma$ are nearly sinusoidal. The site-dependent $Z_{n}, \gamma_{n}$ and $\sigma_{n}$, however, oscillate at a higher frequency. They do so in a non-sinusoidal manner with sharper minima than maxima or vice versa. The phases $\gamma_{n}$ only show small excur-
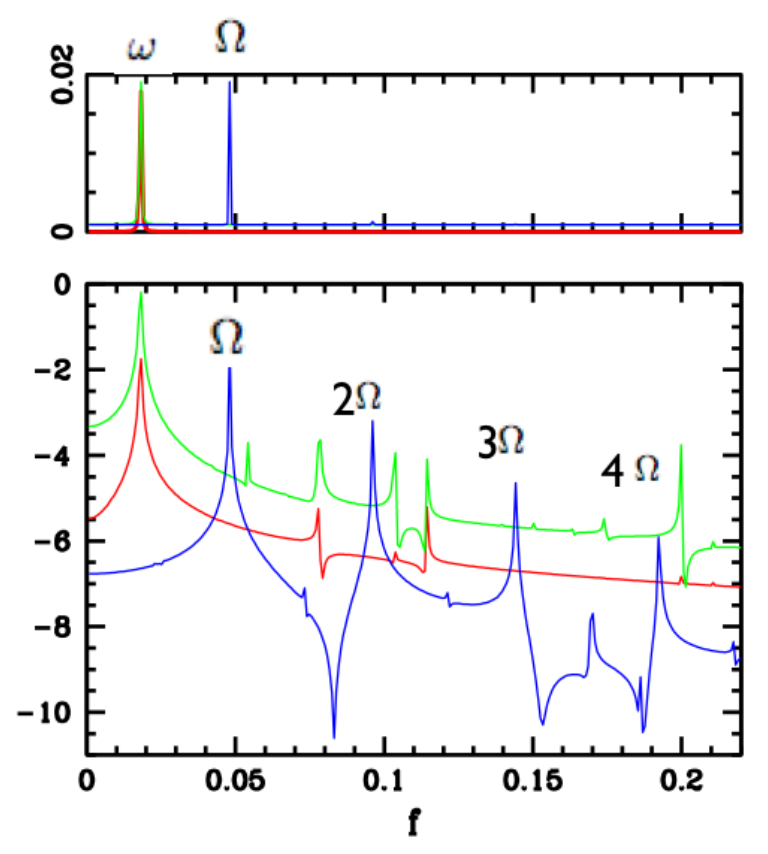

FIG. 3: (color online) Power spectrum or Fourier transform of the time evolution of the spatially-averaged spin population $\rho_{m=+1}$ (red), spatially-averaged spinor phase $\gamma$ (green), and spin-averaged population $\sigma_{n=1}$ at site $n=1$ (blue). The parameters and initial state are as in Fig. 2 and the frequency is in units of $J / \hbar$. The top and bottom panel show the power spectra on a linear and logarithmic scale, respectively. Also indicated are the dominant frequencies $\omega$ and $\Omega$, which have been assigned as due to the spin-dependent and spin-independent interactions, respectively. In the bottom panel higher harmonics of $\Omega$ can be observed.

sions around the average $\gamma$. Finally, we note that the spinor phases are bounded for oscillatory motion.

Figure 3 shows the power spectrum of three of the time traces shown in Fig. 2. It highlights the existence of two dominant frequencies, $\omega$ and $\Omega$, as well as weaker higher harmonics in $\Omega$ indicating non-sinusoidal periodic behavior. The spatially-averaged degrees of freedom predominantly oscillate with a frequency $\omega$, which from simulations with other small $c_{2}$ is found to be proportional to the absolute value of $c_{2}$. In contrast, spin-averaged local populations oscillate nearlysinusoidal with frequency $\Omega$, which from other simulations is found to weakly depend on the spin-changing interaction but is inversely proportional to the number of lattice sites. Finally, the local dynamics for individual spin projections is quasiperiodic with a slow frequency $\omega$ and a faster beat frequency at multiples of $\Omega$. This illustrates the correlations that exist among the spatial and spin components. Detailed studies with various ring sizes indicate that the spinor dynamics is characterized by two frequencies, irrespective of the size of the ring. 


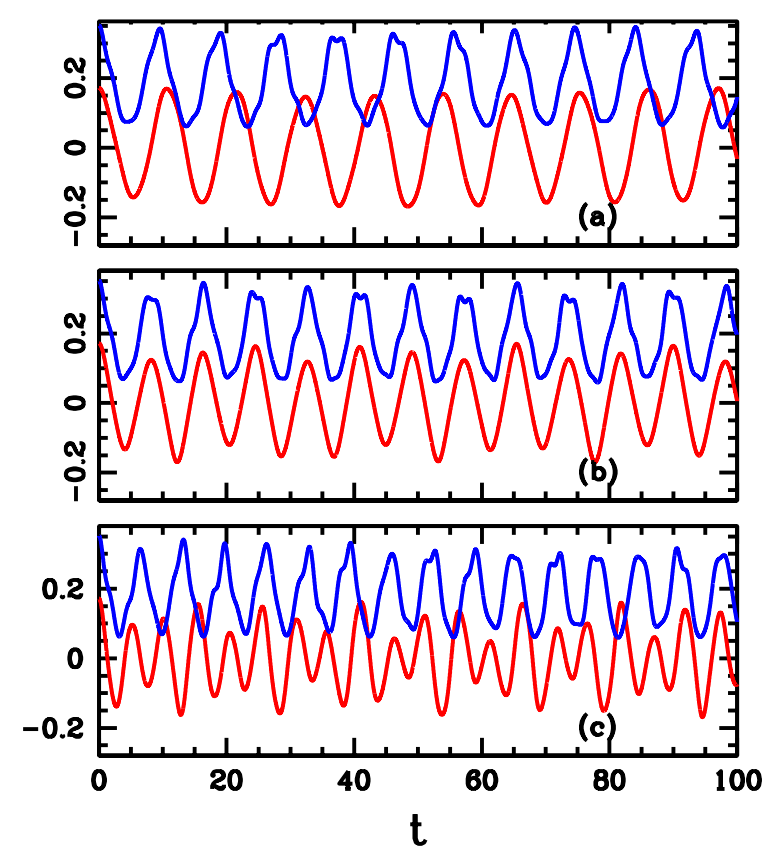

FIG. 4: (color online) Time series for an anti-ferromagnetic spinor showing the resonance condition for increasing spin-changing strength $c_{2}$. Time is in units of $\hbar / J$ and $c_{0}=J$. In all panels $Z$ (red curve) and $\sigma_{n=1}$ (blue curve) are shown. Panels a), b), and c) show results for $c_{2}=0.5 \mathrm{~J}, 0.65 \mathrm{~J}$, and $1.0 \mathrm{~J}$, respectively. The resonance condition occurs for $c_{2}=0.65 \mathrm{~J}$, where the spatially-averaged $Z$ and spin-averaged $\sigma_{n=1}$ oscillate at the same rate. For $c_{2}>0.65 J$ the traces are chaotic.

\section{RESONANCE CONDITION FOR THE ANTI-FERROMAGNETIC CONDENSATE}

Figures 1 and 2 showed that for small $c_{2} / c_{0}$ two frequencies $\omega$ and $\Omega$ are very distinct. For increasing positive $c_{2}$ at fixed $c_{0}$, as shown by time traces in Fig. 4 and power spectra in Fig. 5, both frequencies increase although at a different rate. For a critical spin-changing strength $c_{2}$ when $\omega=\Omega$ the antiferromagnetic spinor reaches a "resonance" state. All three spin-components at all the sites of the ring then oscillate as a single entity. For $c_{0}=J$ this resonance occurs at $c_{2}=0.65 \mathrm{~J}$. For large values of $c_{2}$ the behavior becomes chaotic, which is apparent as broad-band features in the corresponding power spectrum shown in Fig. 5

Fourier analysis of collective as well as local coordinates shows that the resonance state corresponds to matching of not only the dominant frequencies $\omega$ and $\Omega$, but also some of the other less prominent frequencies (not visible in the linear plot) as illustrated in the log-linear plot on the middle row of Fig.5. In fact, at resonance, the dominant or the primary peak is accompanied by secondary satellite peaks, equally spaced on the either side of the primary peak.

Figure 6 further illustrates that at resonance all sites oscillate in phase with same frequency. The spatial profile of the soliton reemerges periodically without any significant change from the initial profile. Thus, the resonance state in an ordered
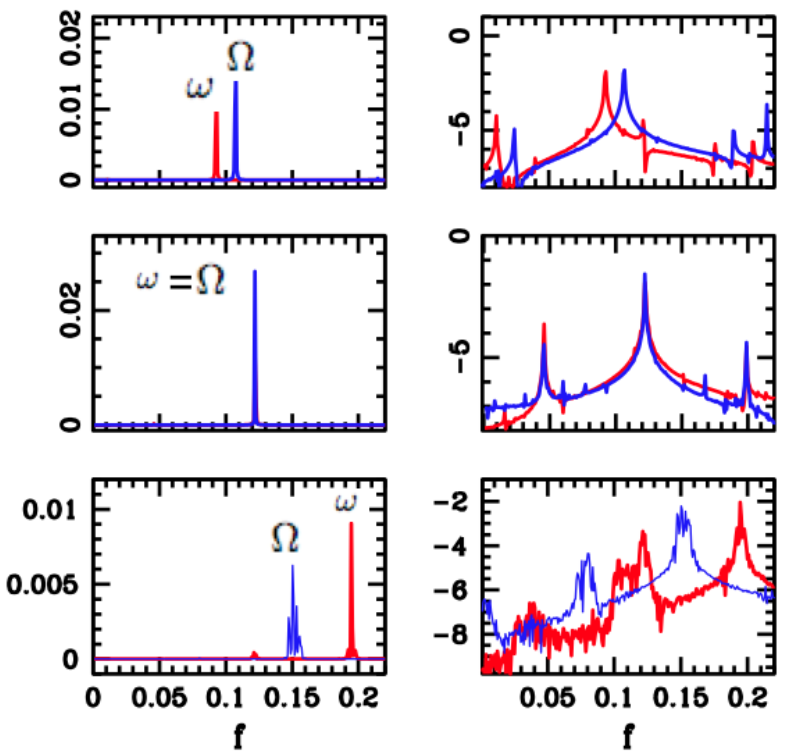

FIG. 5: (color online) Power spectra showing the resonance condition for increasing spin-changing strength $c_{2}$ for the same parameters as in Fig. 4. The frequency is in units of $J / \hbar$. In all panels the spatially-averaged $\rho_{m=+1}$ (red curve) and spin-averaged $\sigma_{n=1}$ (blue curve) are shown. Panels on the left and right show spectra on a linear and logarithmic scale, respectively, while from top to bottom $c_{2}=0.5 \mathrm{~J}, 0.65 \mathrm{~J}$ and $1.0 \mathrm{~J}$.
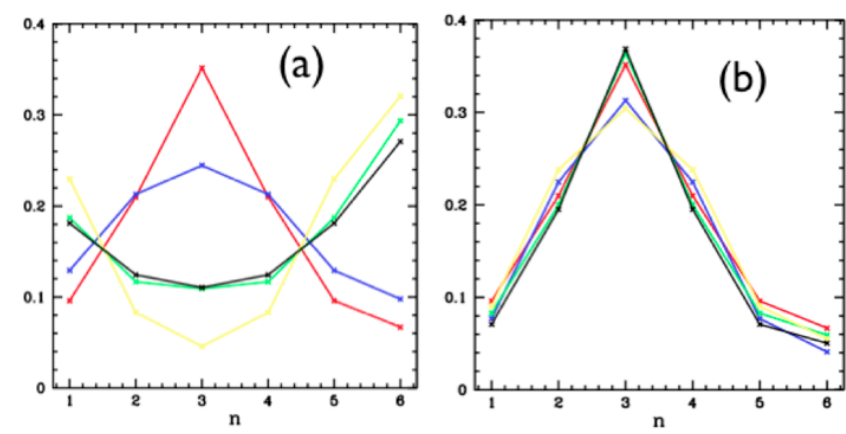

FIG. 6: (color online) Spin-averaged soliton profiles, $\sigma_{n}$, for the antiferromagnetic spinor before and at resonance for five times spaced by time interval $2 \pi / \Omega$, starting with the initial state (red curve). We use $c_{0}=J$ and panels a) and b) correspond to $c_{2}=0.5 \mathrm{~J}$ and $c_{2}=0.65 J$, respectively. The soliton reforms close to its initial state at resonance when $c_{2}=0.65 \mathrm{~J}$.

state where wave functions of all the components of the spinor condensate at all $L$-sites of the ring lattice oscillate in unison and the dynamics is well characterized one frequency. The fact that the soliton profile reemerges periodically provides a unique demonstration of quantum coherence and a novel-type of internal spatio-temporal Josephson effect. 

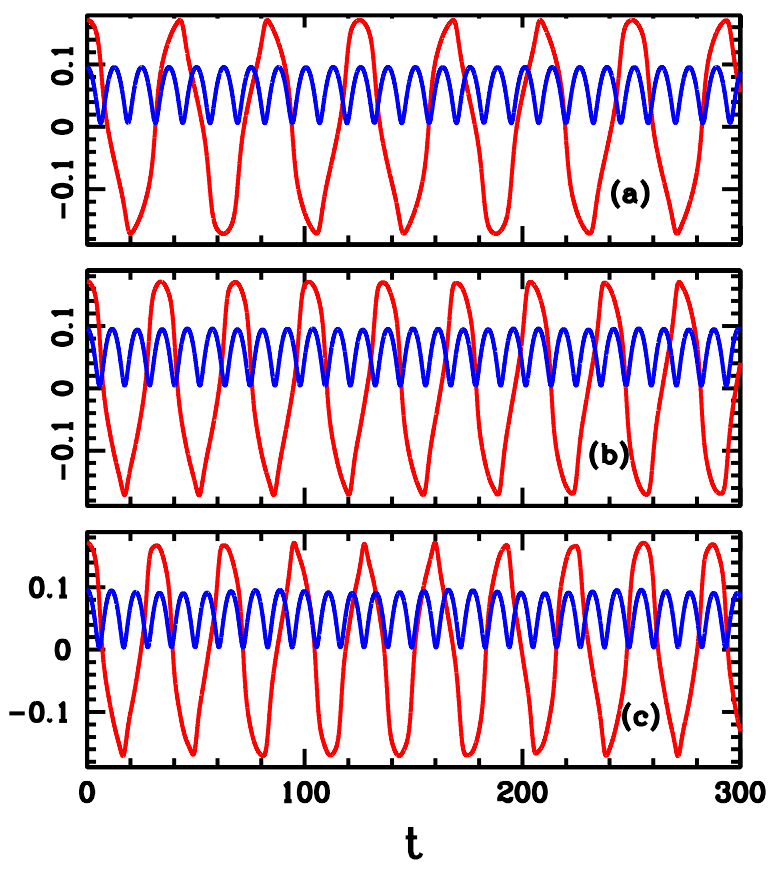

FIG. 7: (color online) Time evolution of spatially-averaged population $Z$ (red) and spin-averaged population $\sigma_{n=1}$ (blue) for a ferromagnetic spinor condensate with negative $c_{2}$. Panel a), b), and c) show traces for $c_{2}=-0.075 \mathrm{~J},-0.09 \mathrm{~J}$, and $-0.095 \mathrm{~J}$, respectively, corresponding to cases before, at, and beyond resonance. We use $c_{0}=J$ and time is in units of $\hbar / J$.
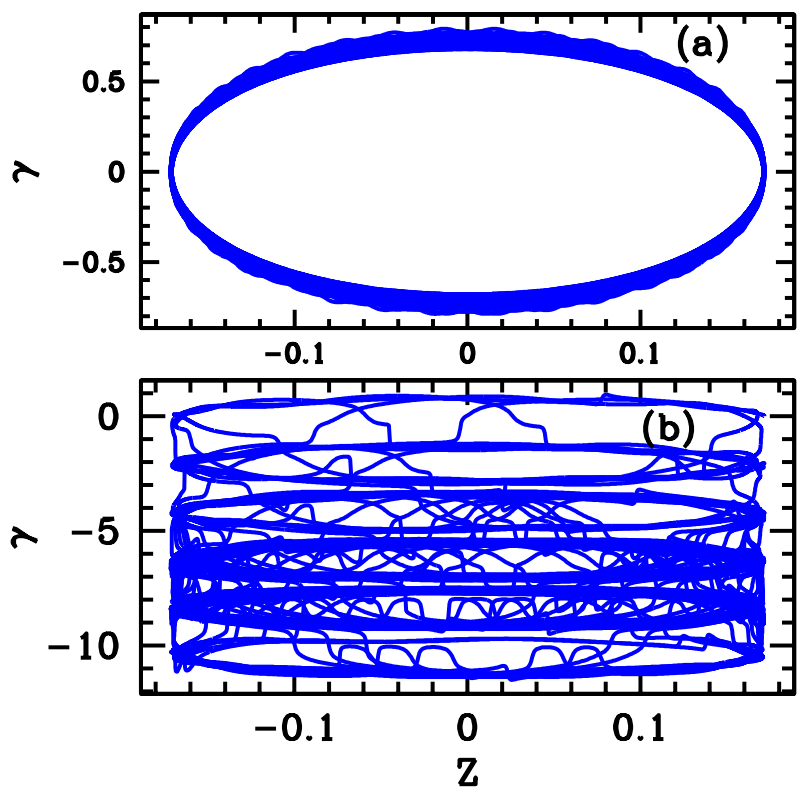

FIG. 8: Phase portraits or parametric plots of the time-evolution of the spinor population $Z$ and phase $\gamma$ for a ferromagnetic spinor at $c_{2}=-0.075 J$, panel a), and $c_{2}=-0.095 J$, panel b). The system is at resonance in panel b). The dynamics in panel b) is accompanied by unbounded motion of the spinor phase. We use $c_{0}=J$.

\section{FERROMAGNETIC CONDENSATE}

We now briefly describe the dynamics of a ferromagnetic spinor condensate with negative $c_{2}$. For a small spinchanging interaction, the dynamics is similar to that of an anti-ferromagnetic spinor. Namely, the spatially-averaged behavior is pendulum-like with dominant frequency $\omega$, while the local population oscillates with frequency $\Omega$. An example is shown in Fig. 7 The local density for each spin component oscillates with both frequencies. A resonance state can again be achieved by increasing $\left|c_{2}\right|$ but, for $c_{0}=J$, now occurs when $\omega / \Omega=3$.

In contrast to the anti-ferromagnetic case, however, the resonant transition is accompanied by unbounded dynamics or phase winding where the phase of the condensate becomes unbounded as shown in Fig. 8 This behavior manifests itself as a zero-frequency mode in a power spectrum. In other words, chaotic dynamics with its broad-band spectrum is accompanied by a transition from an oscillatory to rotational mode for the collective degrees of freedom.

\section{DISCUSSION}

In summary, numerical explorations of mean-field equations of spinor condensate on a ring lattice with a small number of sites reveals strikingly correlated dynamics of the many-body system. Even though the condensate is separated into $L$-sites, soliton dynamics at all sites can be characterized by just two frequencies. With quasiperiodic dynamics at local sites for individual spin components, spatially averaged behavior for each spin component as well as spin-averaged dynamics at each sites is found to be periodic. The fact that the time series describing local dynamics is characterized by two frequencies and these two frequencies untangle in the collective degrees of freedom is rooted in correlations among different spin degrees of freedom at various sites of the lattice. However, proper understanding of these correlations remains an open challenge. Our study with ring lattices of various sizes show that two-frequency characterizations of the spin-1 condensate on a ring lattice is valid irrespective of the number of sites on the lattice.

Our study provides a new illustration of a quasiperiodic route to chaotic dynamics in a many-body system where the critical point is known to be characterized by a resonant state. Simple models of dynamical systems such as the onedimensional circle map [32] are paradigms of quasiperiodic route to chaos, where the critical point corresponds to parameters where the two frequencies are mode-locked. The quasiperiodic route to chaos is a well-established scenario in dynamical systems exhibiting a transition from regular to chaotic dynamics [32]. However, the fact that the critical point describes periodic dynamics is a novelty in many-body systems rooted in the coherence associated with spinor condensate. In this case, the critical point is a highly-ordered manybody state exhibiting a new type of spatial-temporal coherence. At the critical point where the two dominant frequencies are in resonance, the ring lattice with $L$-sites oscillates 
in unison with a single characteristic frequency. This internal Josephson effect where an unscathed soliton profile reemerges periodically provides a novel illustration of both spatial and temporal coherence.

This research is supported by Office of Naval Research, the CONACYT-México and the US Army Research Office.
[1] T. Kuwamoto, K. Araki, T. Eno, and T. Hirano, Phys. Rev. A 69, 063604 (2004).

[2] M.-S. Chang, C. D. Hamley, J. A. Sauer, K. M. Fortier, W. Zhang, L. You, and M. S. Chapman, Phys. Rev. Lett. 92, 140403 (2004).

[3] H. Schmaljohann, M. Erhard, J. Kronjäger, M. Kottke, S. van Staa, L. Cacciapuoti, J. J. Arlt, K. Bongs, and K. Sengstock, Phys. Rev. Lett. 92, 040402 (2004).

[4] J. Guzman, G.-B. Jo, A. N. Wenz, K. W. Murch, C. K. Thomas, and D. M. Stamper-Kurn, Phys. Rev. A 84, 063625 (2011).

[5] A. T. Black, E. Gomez, L. D. Turner, S. Jung, and P. D. Lett, Phys. Rev. Lett. 99, 070403 (2007).

[6] Y. Liu, S. Jung, S. E. Maxwell, L. D. Turner, E. Tiesinga, and P. D. Lett, Phys. Rev. Lett. 102, 125301 (2009).

[7] B. Pasquiou, E. Maréchal, G. Bismut, P. Pedri, L. Vernac, O. Gorceix, and B. Laburthe-Tolra, Phys. Rev. Lett. 106, 255303 (2011).

[8] C. J. Myatt, E. A. Burt, R. W. Ghrist, E. A. Cornell, and C. E. Wieman, Phys. Rev. Lett. 78, 586 (1997).

[9] H. Pu, C. K. Law, S. Raghavan, J. H. Eberly, and N. P. Bigelow, Phys. Rev. A 60, 14631470 (1999).

[10] W. Zhang, D. L. Zhou, M.-S. Chang, M. S. Chapman, and L. You, Phys. Rev. A 72, 013602 (2005).

[11] L. Santos and T. Pfau, Phys. Rev. Lett. 96, 190404 (2006).

[12] R. Barnett, J. D. Sau, and S. Das Sarma, Phys. Rev. A 82, 031602 (2010).

[13] E. Yukawa and M. Ueda, Phys. Rev. A 86, 063614 (2012).

[14] R. Barnett, A. Turner, and E. Demler, Phys. Rev. Lett. 97, 180412 (2006).

[15] A. Widera, F. Gerbier, S. Fölling, T. Gericke, O. Mandel, and I. Bloch, Phys. Rev. Lett. 95, 190405 (2005).

[16] A soliton is a solitary wave with a special collision property, viz., it retains its identity even after collision with another solitary wave. Following a common practice in physics lierature, we will refer to nonlinear localized solitary waves as solitions here.

[17] S. Burger, K. Bongs, S. Dettmer, W. Ertmer, K. Sengstock, A. Sanpera, G. V. Shlyapnikov, and M. Lewenstein, Phys. Rev. Lett. 83, 5198 (1999).

[18] J. Denschlag, J. E. Simsarian, D. L. Feder, C. W. Clark, L. A. Collins, J. Cubizolles, L. Deng, E. W. Hagley, K. Helmerson, W. P. Reinhardt, et al., Science 287, 97 (2000).

[19] R. V. Mishmash, I. Danshita, C. W. Clark, and L. D. Carr, Phys. Rev. A 80, 053612 (2009).

[20] M. Wadati and N. Tsuchida, J. Phys. Soc. Jpn. 75, 014301 (2006).

[21] J. Ieda, T. Miyakawa, and M. Wadati, Laser Physics 16, 678 (2006).

[22] I. I. Satija, R. Balakrishnan, P. Naudus, J. Heward, M. Edwards, and C. W. Clark, Phys. Rev. A 79, 033616 (2009).

[23] A. Smerzi, S. Fantoni, S. Giovanazzi, and S. R. Shenoy, Phys. Rev. Lett. 79, 4950 (1997).

[24] M. Albiez, R. Gati, J. Folling, S. Hunsmann, M. Cristiani, and M. K. Oberthaler, Phys. Rev. Lett. 95, 010402 (2005).

[25] K. W. Mahmud, H. Perry, and W. P. Reinhardt, Phys. Rev. A 71, 023615 (2005).

[26] C. L. Pando L. and E. J. Doedel, Phys. Rev. E 69, 036603 (2004).

[27] P. Buonsante, P. G. Kevrekidis, V. Penna, and A. Vezzani, Phys. Rev. E 75, 016212 (2007).

[28] C. L. Pando L. and E. Doedel, Physica D 238, 687 (2009).

[29] T. Anker, M. Albiez, R. Gati, S. Hunsmann, B. Eiermann, A. Trombettoni, and M. K. Oberthaler, Phys. Rev. Lett. 94, 020403 (2005).

[30] O. E. Alon, A. I. Streltsov, and L. S. Cederbaum, Phys. Lett. A 362, 453 (2007).

[31] C. Pando L. and E. J. Doedel, Phys. Rev. E 71, 056201 (2005).

[32] E. Ott, Chaos in Dynamical Systems (Cambridge University Press, 1993), 1st ed. 